\title{
De « la maleiçon d'Eve en la beneiçon Marie » : enfantements charnel et spirituel dans la correspondance d'Abélard et Héloïse
}

\section{Thibaut Radomme}

\section{(2) OpenEdition \\ Journals}

Édition électronique

URL : http://journals.openedition.org/questes/764

DOI : $10.4000 /$ questes.764

ISSN : 2109-9472

Éditeur

Les Amis de Questes

\section{Édition imprimée}

Date de publication : 15 janvier 2014

Pagination : 67-87

ISSN : 2102-7188

\section{Référence électronique}

Thibaut Radomme, «De « la maleiçon d'Eve en la beneiçon Marie » : enfantements charnel et spiritue dans la correspondance d'Abélard et Héloïse », Questes [En ligne], 27 | 2014, mis en ligne le 15 janvier 2014, consulté le 20 avril 2019. URL : http://journals.openedition.org/questes/764 ; DOI : 10.4000/ questes.764 


\section{De « la maleiçon d'Eve en la beneiçon Marie » : enfantements charnel et spirituel dans la correspondance d'Abélard et Héloïse}

\section{Thibaut RADOMME}

Université catholique de Louvain

La correspondance d'Abélard et Héloïse est traversée par un processus primordial : la transformation d'Héloïse en avatar de pureté, après qu'elle a incarné l'archétype de l'amante. Le corpus épistolaire des deux amants se résume en effet au « dialogue, en somme banal et commun dans la tradition ascétique chrétienne, entre l'âme pécheresse, incarnée par Héloïse, et l'esprit détaché de la matière qui a atteint un stade plus avancé de perfection, autrement dit Abélard ${ }^{1} »$. Ce sont donc la conversion d'Héloïse, son élévation spirituelle et son aptitude à se détacher du péché pour rejoindre le degré de pureté d'Abélard qui constituent le sel et pour ainsi dire le «suspens » de toute la correspondance. Abélard le philosophe met en place plusieurs stratégies (déresponsabilisation d'Héloïse, affirmation forcenée de sa pureté, dépassement psychologique du péché...) pour parvenir à l'achèvement de cette métamorphose, qui doit à la fois servir ses propres intérêts et ceux de la jeune femme - les deux étant intimement liés, comme nous le verrons plus loin. C'est l'une de ces stratégies que nous voudrions analyser plus en profondeur dans la présente étude, celle qui consiste en la promotion de la maternité spirituelle d'Héloïse au détriment

\footnotetext{
${ }^{1}$ Jean-Yves Tilliette, «Introduction », dans Lettres d'Abélard et Hél ‘ise, éd. Éric Hicks et Thérèse Moreau, Paris, Librairie Générale Française, coll. « Le livre de poche », 2007, p. 9-32, cit. p. 29.
} 
de sa maternité physique, à travers l'adoption de la figure de la Vierge Marie comme modèle de vertu. Nous ne procéderons pas à cette enquête à partir de la correspondance latine, mais à partir de sa traduction en ancien français attribuée à Jean de Meun à la fin du XIII ${ }^{\mathrm{e}}$ siècle ( $c a$ 1290) et conservée dans un manuscrit unique de la fin du XIV siècle (Paris, BnF, fr. 920). Version peu étudiée de la correspondance d'Abélard et Héloïse car trop souvent considérée comme un simple décalque du texte latin ${ }^{2}-$, elle mériterait pourtant, par ses qualités littéraires autant que par l'autonomie dont elle fait montre par endroits à l'égard des lettres originales $^{3}$, de recueillir davantage l'attention des chercheurs et spécialistes $\mathrm{du}$ XIII ${ }^{\mathrm{e}}$ siècle français ${ }^{4}$.

${ }^{2}$ Nous renvoyons, pour une analyse de la traduction de Jean de Meun, aux articles suivants : Éric Hicks et Jean R. Scheidegger, «Le corpus abélardien de Jean de Meun : recherches et méthodes ", Bulletin de la Secti 'n de Linguistique de la Faculté des Lettres de Lausanne, 6, 1984, p. 117-145 ; Éric Hicks, « Les métamorphoses du cercle vicieux : inventaires lexicaux et critique textuelle dans un corpus bilingue ", Verbum, numéro spécial, De la plume d' ie à l' rdinateur: études de phil'l'gie et de linguistique 'ffertes à Hélène Naïs, 1985, p. 415-423; Leslie C. Brook, "Synonymic and Near-Synonymic Pairs in Jean de Meun's Translation of the Letters of Abelard and Heloise », Neuphil 'l gische Mitteilungen, 87/1, 1986, p. 1633. D'autres références, traitant de la traduction de Boèce par Jean de Meun, sont utiles à notre propos: Denis Billotte, Le v'cabulaire de la traducti`n par Jean de Meun de la Consolatio Philosophiae de B`èce, Paris, Champion, coll. " Nouvelle Bibliothèque du Moyen Âge», 2000, 2 vol.; Francine Mora, "Du R`man de Phil's 'phie de Simund de Freine au Livre de C'nf" $r$ t de Phil's 'phie de Jean de Meun: évolution des principes et des procédés de la translati', du XII ${ }^{\mathrm{e}}$ au XIII ${ }^{\mathrm{e}}$ siècle », Perspectives médiévales, 26, Translati médiévale. Actes du c`ll que de Mulh use (11-12 mai 2000), dir. Claudio Galderisi et Gilbert Salmon, 2001, p. 51-68.

${ }^{3}$ L'expression «lettres originales » que nous employons est polémique puisque les hypothèses les plus diverses ont été émises à propos de l'authenticité, la paternité et la chronologie de rédaction des corpus latin et français. Cependant, après l'état de la question très exhaustif qu'il propose dans son introduction à la correspondance latine (Jean-Yves Tilliette, "Introduction», dans Lettres d'Abélard et Hél ïse, 'p. cit., p. 20-28), Jean-Yves Tilliette conclut à la solidité de l'hypothèse qui fait aujourd'hui le plus largement consensus : les lettres auraient été écrites en latin au début du $\mathrm{XII}^{\mathrm{e}}$ siècle, sans aucun doute par Abélard et Hélö̈se eux-mêmes, puis elles auraient été compilées dans la première moitié $\mathrm{du} \mathrm{XIII}^{\mathrm{e}}$ siècle à l'instigation de l'abbesse Ermengarde du Paraclet, avant d'être traduites en français par Jean de Meun vers 1290. Pour une vue d'ensemble du débat sur l'historicité des lettres, voir Jacques 


\section{La femme et la maternité au Moyen Âge}

Avant d'ouvrir l'étude de notre texte, il convient de rappeler que la fonction procréatrice est l'unique fonction d'importance que la pensée cléricale reconnaisse à la femme laïque ; c'est donc de la maternité que le sexe féminin tire sa seule légitimité. Claude Thomasset note ainsi :

Les mots retenus pour définir la femme ne servent qu'à évoquer sa fonction principale: il n'est jusqu'à sa faiblesse physique, gage de soumission à l'homme, qui ne favorise la procréation. Cette adéquation et cette réduction à une fonction permettent à tous les théologiens détracteurs de la femme [...] de la considérer comme une force inquiétante, comme un corps qui échappe à la maîtrise d'un esprit, comme un être gouverné par ses organes, particulièrement ses organes sexuels ${ }^{5}$.

Monfrin, «Le problème de l'authenticité de la correspondance d'Abélard et Hélö̈se ", dans Pierre Abélard. Pierre le Vénérable. Les c`urants phil 's 'phiques, littéraires et artistiques en Occident au milieu du XII siècle (C'll que internati 'nal, Abbaye de Cluny, 2-9 juillet 1972), dir. Jean Jolivet et René Louis, Paris, CNRS, coll. «Colloques internationaux du CNRS », 1975, p. 409-424; Peter von Moos, «Le silence d'Héloïse et les idéologies modernes», dans Pierre Abélard. Pierre le Vénérable, 'p. cit., p. 425-468; Hubert Silvestre, "L'idylle d'Abélard et Héloïse : la part du roman ", Bulletin de la Classe des Lettres et des Sciences Mrales et $P ` l i t i q u e s$ de l'Académie R`yale de Belgique, 5' série, 71, 1985, p. 157-200; Barbara Newman, "Authority, Authenticity, and the Repression of Heloise", J'urnal 'f Medieval and Renaissance Studies, 22/2, 1992, p. 121-157; John Marenbon, "Authenticity Revisited», dans Listening $t$ ' Hel ise. The V'ice 'f a Twelfth-Century $W^{\prime}$ man, dir. Bonnie Wheeler, Palgrave, Macmillan, coll. «The New Middle Ages », 2000, p. 19-33.

${ }^{4}$ La Vie et les epistres Pierres Abaelart et Hel ys sa fame. Traducti' $n$ du XIII siècle attribuée à Jean de Meun. Avec une n'uvelle éditi`n des textes latins d'après le ms. Tr'yes Bibl. mun. 802, éd. Éric Hicks, Paris, Champion, coll. « Nouvelle Bibliothèque du Moyen Âge », 1991. Un deuxième tome était prévu mais n'est jamais paru. Nous citons le texte de la correspondance en indiquant le numéro de la lettre, la page et la ligne, comme suit : VI 89, 30.

${ }^{5}$ Claude Thomasset, " De la nature féminine », dans Hist ire des femmes en Occident. $V$ l. 2. Le $M$ yen Âge, dir. Georges Duby, Christiane Klapisch-Zuber et Michelle Perrot, Paris, Plon, 1991, p. 55-81, cit. p. 56. 
La maternité permet de canaliser cette force ${ }^{6}$ en la dotant d'une utilité sociale: dès lors qu'elle est maîtrisée, elle assure en effet la pérennité dynastique et acquiert donc une dignité certaine. C'est ainsi qu'au tournant du XII ${ }^{\mathrm{e}}$ siècle, Marbode de Rennes oppose la putain à la matrone, aux chapitres III (De muliere mala) et IV (De muliere b`na) de son Liber decem capitul 'rum ${ }^{7}$. D’un côté, la «femina blanda ${ }^{8}$ (III, v. 36), comparable à la Chimère, ce monstre proche de la prostituée («ad naturam meretricis ${ }^{9} »$ III, v. 50); de l'autre, la mère : «Nam si desit ager, r' $g$ ', quid tua semina pr'sunt?/Quis queat esse pater, si desit femina

${ }^{6}$ Cette « force inquiétante » est en fait une absence de force, une mollesse, voire une apathie, qui expliquent l'inclination féminine au péché. Le Moyen Âge a hérité cette conception misogyne de l'Antiquité païenne aussi bien que chrétienne : tandis que la théorie hippocratique des humeurs décrit la femme comme froide et humide, c'est-àdire faible, douce, frivole et lascive (par opposition à l'homme dont la nature chaude et sèche induit une force et une stabilité guidées par la raison), Saint Paul explique par la mollesse féminine le fait que le péché originel ait été commis par Ève : «Et ce n'est pas Adam qui fut séduit, mais c'est la femme qui, séduite, tomba dans la transgression » (Première Épître à Timothée, 2, 14). Au VII ${ }^{\mathrm{e}}$ siècle, Isidore de Séville écrit dans ses Etym 'l'giae, XI ii, 17-19 : "Vir nuncupatus, qu`d mai $r$ in e` uis est quam in feminis, unde et uirtus $n$ 'men accepit, siue qu'd ui agat feminam. Mulier uer' a m'llitie, tamquam m'llier, detracta littera uel mutata appellata est mulier. Vtrique enim $f^{\prime \prime}$ rtitudine et inbecillitate c`rp'rum separantur. " (Isidorus Hispalensis, Etym 'l giae, XI. De h`mine et p`rtentis, éd. Fabio Gasti, Paris, Les Belles Lettres, coll. «Auteurs latins du Moyen Âge», 2010, p. 117-119.) : «L'homme (vir) est nommé ainsi, parce que la force (vis) est plus grande en lui que dans les femmes, d'où le courage (virtus) tire aussi son nom, ou peut-être parce qu'il agit envers la femme avec force. Et la femme/femelle (mulier) vient de mollesse ( $m$ `llities), pour ainsi dire femolle ( $m$ 'llier), et une lettre ayant été ôtée ou changée, elle est appelée femme. En effet, tous deux se distinguent l'un de l'autre par la force et la faiblesse respectives de leurs corps. " (nous traduisons). Au XIII ${ }^{\mathrm{e}}$ siècle, on trouve encore, dans la $S^{\prime}$ mme thél'gique du théologien et naturaliste Albert le Grand, une justification de l'explication étymologique d'Isidore par des considérations d'ordre physiologique (Pierre Michaud-Quantin, La psych 'l'gie de l'activité chez Albert le Grand, Paris, J. Vrin, coll. "Bibliothèque thomiste ", 1966, p. 31-32.). Ainsi, la mollesse prêtée aux femmes n'est pas seulement physique, mais elle a une influence décisive sur leurs dispositions morales. L'indolence féminine, traduite sur un plan psychique, justifie l'assertion isidorienne selon laquelle les femmes seraient davantage portées à la licence (Etym `l giae, XI ii, 24).

7 Voir Marbodus Redonensis, Liber decem capitul rum, éd. Rosario Leotta, Roma, Herder, coll. « Biblioteca del Giornale italiano di filologia », 1984, p. 38-45.

${ }^{8}$ « femme enjôleuse, séductrice » (nous traduisons).

9 « à la nature de la prostituée » (nous traduisons). 
mater $^{10}$ ? » (IV, v. 46-47), qui est aussi nourrice, garde-malade et maîtresse de maison s'occupant de la «cura d’mestica ${ }^{11} »(\mathrm{IV}, \mathrm{v}$. 62). La femme se trouve donc valorisée par la maternité, comme le souligne Didier Lett : «Une femme ne peut s'accomplir pleinement que par la maternité qui fait partie intégrante de ses possibilités de rachat. Paul l'exprime clairement: "Néanmoins elle sera sauvée en devenant mère, à condition de persévérer avec modestie dans la foi, la charité et la sainteté" (Première Épître à Timothée, 2,15$)^{12} »$. Ainsi, la sexualité fertile et la maternité s'inscrivent dans l'institution religieuse du mariage, condition de leur acceptabilité sociale: «Puisque la fonction première du mariage est la procréation, l'acte charnel entre les époux est une nécessité. Il est présenté par l'Église comme "une dette conjugale" (debitum c'njugale) que les conjoints se doivent mutuellement ${ }^{13} \gg$. Or Héloïse s'était très fermement refusée à ce qu'Abélard l'épousât après que leur enfant Astrolabe fut né, comme en témoigne son plaidoyer contre le mariage que le philosophe rapporte dans la première lettre de la correspondance, l'Hist' ria Calamitatum (I 14, 384 I 17, 500). Hélö̈se y souligne par exemple les soucis provoqués par un enfant en bas âge et affirme l'incompatibilité de la vie familiale avec les activités intellectuelles du philosophe (I 15, 424). Évidemment, cet argument étant rapporté par Abélard, son authenticité est soumise à caution, d'autant qu'il s'agit là d'une réflexion assez stéréotypée, opposant le premier et le dernier des trois ordres de la société médiévale, celui des 'rat'res et celui, lié à la fonction (re)productrice, des lab 'rat’ res ${ }^{14}$.

\footnotetext{
${ }^{10}$ « Si le champ fait défaut, je te le demande, à quoi bon ta semence ? Qui pourrait être père, si la femme, la mère est absente? » (nous traduisons).

11 « soin domestique » (nous traduisons).

12 Didier Lett, $H^{`}$ mmes et femmes au M'yen Âge. Hist`ire du genre XII $-X V^{e}$ siècle, Paris, Armand Colin, 2013, p. 33.

${ }^{13}$ Ibid., p. 180.

14 Giles Constable, Three Studies in Medieval Religi'us and S'cial Th 'ught, Cambridge, Cambridge University Press, 1995, chap. III, « The Orders of Society », p. 249-360.
} 
Par ailleurs, dans la première lettre qu'elle adresse à Abélard, Héloïse promeut un modèle d'amour pur, vierge de tout calcul ( « je t'aye tousjours embracié par desatrempee amour » II 48, 129), face au déni d'amour d'Abélard, mais aussi face à l'amour feint de certaines épouses :

L'abbesse du Paraclet oppose moins les noms d'épouse et de concubine qu'elle ne déplace les frontières des concepts traditionnels pour faire passer la ligne de partage non plus entre la prostituée et l'épouse, mais au sein de ces deux catégories, entre des épouses et des prostituées « saintes» car désintéressées et douées de la "pudeur de l'âme", et des femmes dont les amours vénales n'ont de cette chasteté véritable que le simulacre, la « continence corporelle ${ }^{15}$.

C'est dans cette perspective qu'il faut comprendre le refus du mariage par Héloïse. La raison fondamentale de ce refus, c'est son amour pour Abélard, comme le précise Étienne Gilson :

[...] il s'agissait surtout pour elle de son amour, c'est-à-dire du tout de sa vie, qui allait être publiquement déshonoré par le projet insensé d'Abélard. Dans la misère morale où elle s'était plongée, Héloïse ne conservait qu'une fierté : celle de cet amour. On conçoit qu'elle pût tenir à la garder intacte et à ne laisser planer nulle équivoque sur le seul sentiment qui lui méritât encore le respect. Précisément, son mariage allait tout compromettre, puisqu' on ne manquerait pas de dire qu'elle s'était laissé séduire par Abélard afin de l'épouser. [...] S'il le fallait, elle serait sa maîtresse, mais, qu'on l'en blâme ou qu'on l'en loue, nul ne pourrait du moins l'accuser d'avoir vendu ce qu'elle avait voulu donner ${ }^{16}$.

Notons qu'Héloïse distingue clairement le mariage de la relation amoureuse : elle n'est pas responsable du premier (dont a découlé

${ }^{15}$ Fanny Oudin, «Héloïse et Abélard. Amants, époux, religieux », Questes, n 20, janvier 2011, p. 38-53, cit. p. 45.

${ }^{16}$ Étienne Gilson, Hél ïse et Abélard. Études sur le M'yen Âge et l'Humanisme [1938], $3^{\mathrm{e}}$ éd. revue, Paris, J. Vrin, 1964, p. 74-75. 
l'émasculation d'Abélard) puisqu'elle s'y est opposée, mais elle est bien responsable de la seconde - et elle est punie de celui-là mais méritait un châtiment pour celle-ci (IV 65, 159). Ainsi, en refusant le mariage institution sociale qui encadre la mutation d'un élan négatif (le caractère hypersexuel féminin) en force positive de cohésion de la communauté (la maternité) -, en ne désirant rien que l'amour ${ }^{17}$, Héloïse se dresse contre les conventions de son siècle et méprise les normes sociales. C'est cet aspect de la figure d'Héloïse que Jean de Meun, successeur de Guillaume de Lorris, retient dans sa continuation du $R^{`}$ man de la $R^{`}$ se (v. 8729-8812 $2^{18}$ ). Le personnage d'Héloïse y revendique en effet une relation parfaitement libre entre l'homme et la femme, loin non seulement des règles du mariage (comme dans la correspondance, où le refus du mariage est, aux yeux d'Héloïse, la seule façon de préserver son honneur en prouvant la pureté de son amour) mais aussi du code extrêmement strict de l'amour courtois ${ }^{19}$ :

${ }^{17}$ Même si elle semble bien consciente du fait que le mariage, en donnant une fin morale à la sexualité, la rend socialement légitime, puisqu'elle écrit : «[nous] convertisimes la laideur de fornicacion pour l'onnour de mariaige » (IV 64, 102).

${ }^{18}$ Guillaume de Lorris et Jean de Meun, Le R`man de la $R$ `se, éd. Félix Lecoy, Paris, Champion, coll. «CFMA », 1965-1970, t. II, p. 16-18.

19 La critique a pu voir, dans le Jean de Meun du $R$ `man de la $R$ `se, un « démystificateur conscient et avisé non seulement de l'ordre social et religieux mais, plus encore, de l'idéal courtois et des mœurs » (Rita Lejeune, "La femme dans les littérature française et occitane $\mathrm{du} \mathrm{XI}^{\mathrm{e}}$ au $\mathrm{XIII}^{\mathrm{e}}$ siècle $»$, Cahiers de civilisati $n$ médiévale, 20/78-79, 1977, p. 201-217, cit. p. 214). Sans aller jusqu'à la lecture indéniablement anachronique d'un hédonisme communiste (Jean-Charles Payen, La $r$ 'se et l'ut'pie. Rév'luti'n sexuelle et c'mmunisme $n$ 'stalgique chez Jean de Meung, Paris, Éditions sociales, coll. «Classiques du peuple. Critique», 1976), il

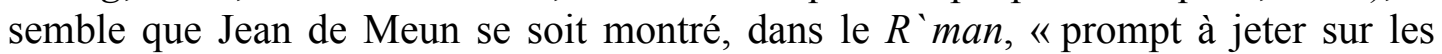
choses de l'amour un regard cynique et grivois, bien éloigné de l'idéalisme courtois » (Michel Zink, Littérature française du M yen Âge [1992], Paris, PUF, coll. "Quadrige», 2004, p. 244). La polémique sur l'emploi du mot c'uilles par Raison (v. 6913-7154), la métaphore obscène des reliques religieuses (v. 2118521246), la promotion par Genius d'une morale sexuelle naturaliste, par opposition à l'éthique mortifère de l'abstinence courtoise (v. 20597-20652), et bien entendu la scène finale, assez explicite, de la capture de la rose (v. 21645-21749) sont autant d'épisodes qui témoignent d'une réorientation radicale du projet courtois de Guillaume de Lorris sous la plume de Jean de Meun. Jean de Gerson, qui prend part, aux côtés de Christine de Pizan, à la querelle du $R$ `man de la $R$ `se au tout début du $\mathrm{XV}^{\mathrm{e}}$ siècle, écrit d'ailleurs à propos de Jean de Meun, dans son Traité c'ntre le 
Et [Héloïse] requeroit que il l'amast, mes que nul droit n'i reclamast, fors que de grace et de franchise, sanz seigneurie et sanz mestrise ${ }^{20}$

Le vers 8752 est également très intéressant: Héloïse veut qu'Abélard puisse étudier «touz siens, touz frans, sanz soi lier». Le personnage du mari jaloux (dont les propos sont rapportés par la personnification $\mathrm{d}^{\prime} \mathrm{Ami}^{21}$ ) ne mâche d'ailleurs pas ses mots : « Mariages est maus liens » (v. 8803).

\section{Promotion de la maternité spirituelle}

La maternité, envisagée dans le cadre du mariage, est donc primordiale pour la femme du Moyen Âge: elle constitue une voie privilégiée d'accomplissement social. Pourtant, Abélard rejette cette maternité avec violence. Il en avait déjà arraché Héloïse lorsque cette dernière avait donné naissance à leur fils. Ainsi, comme il l'explique dans l'Hist'ria Calamitatum, Abélard déguise Héloïse en religieuse, c'est-à-dire en vierge, alors qu'elle est enceinte et l'envoie loin de Paris, près de Nantes, afin d'accoucher dans le plus grand secret :

En une nuit adoncquez que son oncle n'estoit mie a l'ostel, si comme nous avions ensemble pourparlé avant, je la portay par larrecin hors de

$R$ `man de la $R$ `se: «[...] a ung commencement qui par aventure se porroit assés passer selond son fait - mesmement entre Crestiens -, il adjousta tres orde fin et moien desraisonnable contre Raison » (Le Débat sur le R 'man de la R 'se, éd. Éric Hicks, Paris, Champion, coll. « Bibliothèque du XV siècle », 1977, p. 85, 1. 664).

${ }^{20}$ Guillaume de Lorris et Jean de Meun, Le R`man de la $R$ `se, éd. cit., p. 17, v. 87478750.

${ }^{21}$ Le $R$ `man de la $R$ `se de Jean de Meun est en effet constitué, à la façon des poupées gigognes, d'un enchevêtrement resserré de discours subordonnés à la voix du narrateur (l'amant, c'est-à-dire le jeune homme qui s'est endormi et a fait un rêve qu'il veut mettre en vers : « Or veil cel songe rimeer », v. 31). Ainsi, Ami apporte au jeune homme la consolation de son expérience en un long monologue (v. 72049972), au cours duquel il dénonce, entre autres, la perversion des mœurs féminines. Un personnage est mis en scène dans le discours d'Ami, celui du mari jaloux, qui accable sa femme d'un violent discours misogyne (v. 8437-9330). C'est dans ce discours du mari jaloux, rapporté par Ami, dont le propos est lui-même rapporté par le narrateur, que prend place la référence à Abélard et Héloïse. 
sa maison et l'envoyay tantost en mon païs : la demoura elle tant avec une moye sueur que elle ot ung enfant malle, et li mist nom Astralabius. (I 13, 356)

À peine mis au monde, l'enfant est soustrait à sa mère et confié à la sœur d'Abélard : «Quant noz petit enfes fu nés, il fut bailléz a ma sereur. » (I 17, 508). Le mariage lui-même, symbole de la sexualité socialement légitime c'est-à-dire de la maternité, est conclu secrètement, en pleine nuit, et la séparation des corps suit immédiatement la cérémonie, excluant toute possibilité de nouvelle consommation - ou de consommation publique du moins, qui entraine l'enfantement, n'autorisant donc que la sexualité socialement illégitime des plaisirs clandestins et bannissant de fait l'accomplissement maternel de la femme ${ }^{22}$.

En lieu et place de la maternité physique, qu'il condamne sans appel, Abélard promeut la maternité spirituelle à travers un plaidoyer violent et passionné dans la lettre $\mathrm{V}$ :

Mes li besans de ta saigesce, com grans usures raporte il chascun jour a Nostre Seigneur, qui as ja enfanté a Nostre Seigneur maintes filles esperituelles, endementres que je suis brehaings $\mathrm{du}$ tout et me travaille en vain es filz de perdicion! Or comme ce fust escomeniable dommaige ! comme ce fust plorable perte, se tu entendoies aus ordures des deliz charnelz et enfantasses en doleur au monde pou de filz, qui orendroit enfantes a joie montepliable lignee au ciel! Ne tu ne feusses pas neis plus que femme, qui sourmontes orendroit les hommes et qui as tournee la maleiçon d'Eve en la beneiçon Marie. O comme honteusement icelles sacrees mains, qui reversent ore les volumes devins, servissent aus

${ }^{22}$ Ainsi, Abélard retrouve Héloïse dans le secret du monastère d'Argenteuil où il l'avait fait placer après leur mariage, et les époux s'adonnent là aux plaisirs de la chair, « en lieu de si grant reverence et qui estoit consacréz a la Souveraine Vierge » (V 78, 297). La clandestinité d'une relation sexuelle pourtant autorisée éclabousse donc de l'opprobre du blasphème la virginité souveraine de Marie, à laquelle les lieux sont consacrés. 
ordures des besoignes femenines ! (V 82, 424)

Abélard établit une opposition très nette entre la maternité physique et la maternité spirituelle. Il a des mots très durs contre la première, semblant d'ailleurs occulter complètement le fait qu'Héloïse et lui sont les parents bien réels d'Astrolabe, mais aussi contre les tâches de la maîtresse de maison et la sexualité humaine en général (ces deux derniers éléments sont désignés par le même mot extrêmement dépréciatif : «ordures »). Il fait référence au récit de la Genèse et à la malédiction de l'enfantement dans la douleur, à la suite du Péché originel : « Il [Dieu] dit à la femme : "Je ferai qu'enceinte, tu sois dans de grandes souffrances; c'est péniblement que tu enfanteras des fils" »(Genèse 3, 16). Enfin, en détournant Héloïse des tâches matrimoniales, Abélard l'éloigne du troisième ordre de la société médiévale pour la pousser vers le premier, celui des hommes qui prient nous verrons que cela aura son importance. Tout au contraire, Abélard célèbre la maternité spirituelle d'Héloïse, qui lui fournit une « montepliable lignee au ciel » (c'est-à-dire, littéralement, «qui a la faculté de se multiplier, infinie ») en tant qu’épouse du Christ (sp `nsa Christi) plutôt que d'Abélard, dans l'amour céleste plutôt que terrestre. Il affirme la pureté d'Héloïse en contraste avec sa propre infamie, puisqu'étant stérile («brehaings »), il n'a pour sa part aucune descendance spirituelle ${ }^{23}$, sauf

${ }^{23} \mathrm{~L}$ 'on pourrait considérer qu'Abélard, en fondant le Paraclet et en le dotant d'une règle monastique, donne lui aussi naissance à une descendance spirituelle. C'est vrai en un sens. Cependant, c'est Héloïse surtout qui souligne le rôle d'Abélard dans la fondation du monastère (voir par exemple le dernier paragraphe de la lettre VI 106, 626) et c'est elle encore qui désigne le philosophe du nom de père (ainsi, l'adresse de sa première lettre commence par ces mots : «A son seigneur, mes a son pere » II 45, 3). Au contraire, Abélard, même s'il se lamente sur sa "vie sans proufit et chetive » qui ne produit pas de " fruit» (I 36, 1168) avant de narrer la création du Paraclet, souligne le fait qu'il n'y a joué un rôle que de peu d'importance. Ainsi, ce n'est pas lui qui a développé le modeste oratoire en florissante abbaye, mais le Paraclet luimême, c'est-à-dire le Saint-Esprit : « Mes le vray Paraclit mesmes [...] pourvit a ce propre oratoire si comme il devoit. » (I 36, 1184). Il affirme plus loin que les religieuses se sont «plus montepliéz en ung an que en cent se je eusse illec demouré » (I 37, 1205), car la pauvreté de femmes seules émeut davantage les 
ces «filz de perdicion», moines de Saint-Gildas-de-Rhuys dont il est devenu l'abbé vers 1127, qui veulent sa mort et lui causent tant de $\operatorname{souci}^{24}$. En prenant le voile religieux, Héloïse a en effet épousé le Christ: «Beneurés est li changes de telz noces, par quoy en avant femme d'un chetif home, soies ore eslevee es chambres du souverain roy » $(\mathrm{V} 71,33)^{25}$. Les adresses des lettres d'Abélard semblent devoir le rappeler à une Héloïse oublieuse : «A Heloys sa tres amee sereur en Jhesu Crist » (III 54, 2) devient ainsi «A l'espouse de Jhesu Crist » (V 70, 3) - le mémento se double d'une froide mise à distance de celle qui s'espérait encore l'amante d'Abélard, traduite par la disparition du groupe qualificatif « tres amee ». Bien entendu, l'analogie filiale est un lieu commun de la pensée monastique. Abélard en rend compte dans l'Hist ria Calamitatum : «mes filz - c'est de mes moines -, qui me sont bailliés comme a leur abbé - c'est a dire comme a leur pere» (I 41, 1366). Mais la maternité spirituelle promue par Abélard va beaucoup plus loin que ce $t{ }^{`}{ }^{`} s$, puisqu'elle fait d'Héloïse une "plus que femme», qui surpasse les hommes, dont les mains sont « sacrees » et qui « as tournee la maleiçon d'Eve en la beneiçon

bonnes âmes et les incline à la charité. Il précise enfin qu'il fallut « que tuit leurs voisins me blamassent forment de ce que je ne metoie pas conseil en leur povreté tant come je peusse et deusse » $(\mathrm{I} 37,1219)$ pour qu'il se résolve à les rejoindre et à leur apporter son soutien. On voit donc qu'Abélard minimise très nettement son rôle dans la fondation du Paraclet. Par ailleurs, il réaffirme à plusieurs reprises qu'Héloïse et lui-même sont frère et sœur, et non père et fille (ainsi que le précise l'adresse de la lettre III : «A Heloys sa tres amee sereur en Jhesu Crist, Pierre Abayelart, ses freres en icelui mesmes » III 54, 2). Il nous semble qu'il faut voir dans cette modestie inaccoutumée un renforcement de l'opposition entre la fertilité spirituelle d'Héloïse et sa propre infertilité.

${ }^{24}$ L'emploi du verbe « travaille » renvoie en effet à l'idée de peine, de tourment, voire de souffrance, comme le suggère son origine étymologique du bas latin tripalium (Französisches Etym 'l'gisches Wörterbuch XIII-2, 287b et 291b).

${ }^{25}$ Niant son amour pour Héloïse ( $«$ Car je estoie acoupléz a toy par si grant ardeur de charnel delit » V 80, 350), Abélard y substitue « l'amour du celestial espous » (V 72, 83). Il dément l'amour terrestre et valorise l'amour céleste, en opposant les mariages humain et divin : "Cil [Jésus-Christ] t'amoit vrayement, non mie moy. M'amour, qui l'un et l'autre envelopoit en peschiéz, devoit estre appellee convoitise charnel, non pas amour. Je acomplissoie en toy mes chetis delis, et c'estoit quantquez je amoye » (V 84, 511). 
Marie » (V 82, 431). On retrouve là l'idée du jeu médiéval sur les mots Eva - Ave (Maria), signifiant que Marie ouvre aux hommes la voie du salut par le rachat du péché dans lequel Ève les avait plongés, à la fois en sacrifiant son Fils pour la rédemption de l'humanité et, en tant que mère consolatrice de tous les pécheurs, en intercédant pour eux auprès de son Fils $^{26}$. Ève et Marie incarnent donc les deux figures féminines les plus radicalement opposées, comme le rappelle saint Jérôme dans une formule célèbre de son épître XXII : «m`rs per Evam, vita per Mariam ${ }^{27} »$. L'opposition entre femme-putain et femme-mère, que nous rapportions cidessus et qui s'ancre dans la perspective laïque de l'enfantement physique, est ainsi transcendée en l'opposition de la femme-putain avec la viergemère, dans la pensée mystique de l'enfantement spirituel, qui autorise la maternité sans compromettre la virginité.

\section{La Vierge Marie comme modèle de maternité}

Abélard rejette donc le mariage terrestre et la maternité physique au profit du mariage céleste et de la maternité spirituelle, condamnant simultanément la sexualité et l'amour humain. Héloïse est ainsi appelée à incarner la figure paradoxale de Marie, mère mais vierge. Alors que le dogme de la conception virginale du Christ rend compte de la virginité de

\footnotetext{
${ }^{26}$ Ainsi, la fonction médiatrice de Marie dans la religion du recours est clairement soulignée par des prières telles que le Sub tuum praesidium, ou le Salve, Regina, parfois attribué au moine Hermann Contract au XI ${ }^{\mathrm{e}}$ siècle. Le thème de la Vierge au grand manteau, protégeant des pans de son habit les hommes frappés par la colère de Dieu, prospère singulièrement $\mathrm{au} \mathrm{XIV}{ }^{\mathrm{e}}$ siècle, dans le climat de peur provoqué par l'épidémie de peste noire de 1348. Toutes ces représentations particulières témoignent du rôle d'avocate des chrétiens et de suppliante auprès de Dieu qui est confié à la Vierge. Voir Jean Delumeau, Rassurer et Pr`téger. Le sentiment de sécurité dans l'Occident d'autref'is, Paris, Fayard, 1989, p. 261-289.

27 «la mort par Ève, la vie par Marie» (nous traduisons). Hieronymus, Epistulae, éd. Isidorus Hilberg, Vindobonae, Tempsky, coll. «Corpus Scriptorum Ecclesiasticorum Latinorum », 1910, p. 173.
} 
$\mathrm{Marie}^{28}$, c'est l'élaboration intellectuelle du principe de maternité spirituelle qui dote Héloïse de la virginité dans sa deuxième vie matrimoniale. L'Imitati`Christi se fait ainsi Imitati`Mariae : le modèle qu'Abélard propose à Héloïse n'est pas Jésus-Christ, mais Sa mère. Il faut bien noter qu'il s'agit d'une imitati et que, au même titre que l'imitation parfaite du Christ est un but inaccessible pour le chrétien, la Vierge est pour Héloïse un modèle à suivre mais non à atteindre dans son unicité souveraine: " "Seule, sans exemple, vierge et mère Marie", comme l'affirment divers recueils carolingiens ${ }^{29} »$. Le propos d'Abélard est d'offrir à Héloöse un modèle, non de la représenter en Vierge Marie dans la correspondance. Ainsi, contrairement à ce que suggère Juanita Ruys dans l'article qu'elle consacre à la maternité d'Héloïse ${ }^{30}$, il ne nous semble pas qu'Abélard mette Héloïse à la place de la Vierge lorsqu'il l'invite à pleurer la mort du Fils unique de Dieu. Juanita Ruys écrit en effet: «just as Abelard in Ep. 5 recasts Christ as Hel'ise's true sp 'use in place 'f himself, $s$ ' in like manner he recasts Christ as Hel'ise's 'ne true s' $n$ and 'nly pr`per `bject `f maternal pity in place `f Astr`labe ${ }^{31} »$. Si le remplacement d'Abélard par le Christ comme véritable époux d'Héloïse est évident, l'hypothèse que l'amour maternel d'Héloïse doive substituer le Christ à Astrolabe nous semble erronée. Certes, le passage de la correspondance

${ }^{28}$ Pour un historique des débats théologiques ayant présidé à la formation de la doctrine de la virginité mariale «ante partum, in partu, p st partum» («avant, pendant et après l'accouchement »), voir Jacques Dalarun, «La Madeleine dans l'Ouest de la France au tournant des $\mathrm{XI}^{\mathrm{e}}-\mathrm{XII}^{\mathrm{e}}$ siècles ", Mélanges de l'Éc 'le française de $R$ `me. M`yen Âge, Temps M dernes, 104/1, 1992, p. 71-119, cit. p. 90-105.

${ }^{29}$ Jacques Dalarun, « Regards de clercs », dans Hist ire des femmes en Occident. V'l. 2. Le M'yen Âge, 'p. cit., p. 31-54, cit. p. 39.

${ }^{30}$ Juanita Feros Ruys, "Quae maternae immem `r naturae : the Rhetorical Struggle over the Meaning of Motherhood in the Writings of Heloise and Abelard ", dans Listening $t$ 'Hel'ise, 'p. cit., p. 323-339.

${ }^{31}$ Ibid., p. 326 : « tout comme Abélard, dans la lettre V, établit le Christ à sa propre place comme véritable époux d'Héloïse, de la même façon il érige le Christ en véritable fils unique d'Héloïse et seul objet approprié de compassion maternelle à la place d'Astrolabe » (nous traduisons). 
que Juanita Ruys cite pour appuyer son propos invite Hélö̈se à pleurer la mort de Jésus comme on pleure celle d'un fils unique, mais il est suivi des mots suivants, que l'auteur ne rapporte pas, et qui prouvent bien qu'Héloïse ne doit pas pleurer le Christ comme une mère, mais comme une épouse :

Regarde de quel plaint la mesniee, et quel pleur toute la court soit degastee, et quant tu seras venue a l'espouse de ce seul filz, tu ne soustendras pas ses brais qui ne sont pas souffrables. Suer, si soit tes plains; si soit tes brais : qui par beneuré mariaige te es couplee a tés espous (V 84, 493).

Héloöse est donc considérée comme l'épouse du Christ et non comme sa mère, mais à l'instar de la mère du Christ, elle est appelée à protéger les hommes de la douceur de sa maternité : c'est donc la descendance spirituelle d'Héloïse qui se substitue à Astrolabe dans le rôle du fils aimé, et non le Christ ainsi que le prétend Juanita Ruys. Comme la Vierge Marie, Hélö̈se est appelée à devenir la mère de tous les hommes (ce qu'autorise la maternité spirituelle, qui fournit une « montepliable lignee ») et à prier pour leur salut. Abélard note d'ailleurs que le pouvoir de la prière des femmes, et des mères en particulier, est depuis longtemps bien connu : «li Vieulx Testament racontz que deux mors furent resuscitéz aus prieres de leurs meres » (III 57, 100). Héloïse doit prier pour l'humanité, et plus particulièrement pour Abélard, pour qui elle peut obtenir davantage du Christ en tant qu'elle est Son épouse :

Or ne te merveille pas doncquez se jé, et a ma vie et a ma mort, me commant a voz oroisons especialment, comme certaine chose soit par droit commun que les espouses peuent plus vers leurs seigneurs en priant que leurs mesnies, et les dames plus que li sers (V 71, 36)

Exactement comme la Vierge (et les saints), Héloïse reçoit d'Abélard la mission délicate d'exercer son pouvoir d'intercession. En tant que mère spirituelle (et non physique) de ses filles mais aussi de tous les hommes, en 
tant qu'épouse du Christ - parce qu'elle peut dès lors obtenir davantage de ce dernier ${ }^{32}-$, Héloïse est appelée à intercéder, par la prière, pour le salut de leurs âmes : ici il ne s'agit pas d'obtenir la résurrection comme dans l'Ancien Testament, mais la vie éternelle après la mort, celle qu'Abélard craint pour lui-même : «Et se il avenoit que Nostre Sire me baillast es mains de mes anemis - c'est assavoir que il feussent plus fort ou que il m'occissent, ou que je muire par quelcunquez cas loing de vous » (III 59, 183). Telle est la « beneiçon Marie ».

\section{Une imitatio doublement problématisée}

L'imitation de la Vierge s'inscrit pleinement dans le contexte d'un important essor du culte marial dans tout l'Occident aux XI $\mathrm{II}^{\mathrm{e}}$ et $\mathrm{XII}^{\mathrm{e}}{ }^{\mathrm{e}}$ siècles ${ }^{33}$, « qui traduit l'affirmation de la prévalence, dans la société, des modèles spirituels et de l'idéal de la virginité, par essence contraire à la nature

${ }^{32}$ C'est tout le paradoxe de la situation d'épouse du Christ qui est celle d'Héloïse : d'un côté sa prière en est plus efficace, de l'autre son péché en est plus grave. En effet, lorsqu'Abélard et Héloïse ont des relations sexuelles au couvent d'Argenteuil (V 78, 292-300), même si Héloïse n'est encore que la future épouse de Jésus (elle réside déjà au couvent mais n'a pas encore pris le voile monastique ; elle ne le fera qu'après l'épisode de l'émasculation: "Ambedui donquez receusmes sacré habit, jé en l'abbaie de Saint Denis, et elle au moustier d'Argentueil dessus dit » I 19, 573), leur faute se trouve teintée d'une coloration d'adultère. Héloïse s'étonne d'ailleurs du fait que le châtiment subi par Abélard soit ordinairement réservé aux hommes convaincus d'adultère, et qu'Abélard en ait été accablé dans le mariage : "Certes cele payne que tu souffris fust asséz a homme pris en toutes manieres d'avotire; et ce que li autre deservent par avouture, tu encoureus et comparas par mariaige [...]. Et ce que les femmes avoutres font a leurs houlliers, ta femme le t'a fait » (IV 64, 106). Ainsi, « En exhortant Héloïse à aimer Jésus, son véritable époux, plus que lui-même, Abélard rachète en fait l'adultère physique commis contre Dieu par un "adultère spirituel" dont le philosophe est désormais la victime volontaire. » (Didier Lett, 'p. cit., section 12.1.4).

${ }^{33}$ Voir Claire Thiellet, « La dévotion mariale en Occident autour de l'an Mil », dans La Dév 'ti $n$ mariale de l'an mil à $n$ 's j’urs, dir. Bruno Béthouart et Alain Lottin, Arras, Artois Presses Université, coll. "Histoire», 2005, p. 75-80 ; Daniel Russo, «Les représentations mariales dans l'art d'Occident. Essai sur la formation d'une tradition iconographique ", dans Marie. Le culte de la Vierge dans la s'ciété médiévale, dir. Dominique Iogna-Prat, Éric Palazzo et Daniel Russo, Paris, Beauchesne, 2005, p. 173-291 ; François-Jérôme Beaussart, "La littérature mariale au Moyen Âge », dans La Dév ‘ti n mariale de l'an mil à n`s j’urs, 'p. cit., p. 301-312. 
charnelle de la femme ${ }^{34} »$. L'imitation est pourtant doublement problématisée, d'abord à cause de l'effronterie d'Héloïse, qui résiste à l'entreprise de déresponsabilisation mise en place par Abélard en affirmant, dans les lettres II et IV, sa responsabilité aussi bien que sa culpabilité (mais cette effronterie se résout dans le silence de la lettre $\mathrm{VI}^{35}$ ), ensuite parce que la virginité d'Héloïse est évidemment manquée du fait de sa liaison charnelle avec Abélard. Cependant ce dernier, ayant été émasculé et donc privé de l'organe nécessaire à l'accomplissement de sa tâche maritale, est symboliquement mort sur le plan sexuel et en tant qu'époux : l'instrument de la défloraison a disparu. Par ailleurs, le philosophe voit en sa mutilation non pas une punition divine mais un moyen de guérison, l'émasculation ayant eu une vertu thérapeutique pour les âmes des deux amants. Il faut rappeler ici que la mutilation est considérée comme une humiliation terrible au Moyen Âge car, originellement appliquée aux criminels ayant troublé l'ordre public, elle devient un signe physique de félonie ${ }^{36}$. Plus particulièrement dans le contexte chrétien, elle est la trace corporelle du péché :

[It] calls to mind certain passages from the New Testament, such as the words of Christ in Matthew 5:30: «And if your right hand causes you to sin, cut it off and throw it away; it is better that you lose one of your members than that your whole body go into hell ». Here we have a

${ }^{34}$ Christiane Klapisch-Zuber, «Femme », dans Dicti nnaire du M yen Âge, dir. Claude Gauvard, Alain Libera et Michel Zink, Paris, Presses Universitaires de France, coll. « Grands Dictionnaires », 2002, p. 520.

${ }^{35}$ Héloïse entame son épître par ces mots, signifiant ainsi sa soumission aux projets d'Abélard : "Pour ce que tu ne me puisses reprandre par aventure en aucune chose de m'obedience, le frain de ton commandement est mis aus parolles de ma desatrempee douleur » $(\mathrm{V} 88,3)$. À propos du silence d'Héloïse, voir l'article de Peter von Moos « Le silence d'Héloïse et les idéologies modernes », art. cit.

36 Voir David S. King, "The Meaning of Amputation in the Chans `ns de geste ", Symp sium, 62/1, 2008, p. 35-52. Voir particulièrement la note 15, sur l'origine historique de la tradition de mutilation des félons. 
rapprochement of sin and dismemberment ${ }^{37}$.

La honte que représente la mutilation, cette «trop cruelle et [...] trop honteuse venjance » (I 18,532), est clairement exposée par Abélard dans l'Hist'ria Calamitatum. Rapportant que son entourage se lamentait à propos de sa blessure, il commente en ces mots : «je estoie plus blecié de la pitié que j'avoie d'eulx que du tourment de ma plaie ; et plus me grevoit la honte que la plaie, et plus estoie tormentéz de honte que de doulour » (I 18, 545). Alors que lui reviennent en mémoire sa gloire passée et les actions qui l'ont mené là (la mutilation étant le signe du péché, il est normal qu'elle ravive chez Abélard le souvenir des fautes), songeant à la douleur que sa propre plaie infligera à ses proches, il conclut :

De ce neis ne me confondoit pas peu que selonc la lettre ocierre de la Loy, la vindication et li destruimens des escouilléz est si granz qu'a ceulx qui les coillons ont copéz ou escorchiéz est deffendue l'entree des eglises comme hommes puans et ors (I 18, 559).

La mutilation est donc honteuse parce qu'elle est l'expression corporelle du péché. C'est parfois le cas également de la maladie ${ }^{38}$. Que l'on songe par

${ }^{37}$ Ibid., p. 38 : « [Cela] rappelle certains passages du Nouveau Testament, tels que les mots du Christ dans Matthieu, v, 30 : "Et si ta main droite entraîne ta chute, coupe-la et jette-la loin de toi : car il est préférable pour toi que périsse un seul de tes membres et que ton corps tout entier ne s'en aille pas dans la géhenne". Ici nous avons un rapprochement du péché et du démembrement » (nous traduisons).

${ }^{38} \mathrm{La}$ maladie peut, au contraire, représenter une marque d'élection : elle peut être une épreuve infligée par Dieu qui prélude à la régénération et donc au salut. Joinville raconte ainsi que saint Louis décida de partir en croisade après être tombé gravement malade et avoir été guéri par Dieu (Jean de Joinville, Vie de saint L ‘uis, éd. Jacques Monfrin, Paris, Librairie Générale Française, coll. «Le livre de poche », 1995, p. 210, § 106-107). La maladie conduit aussi à la sainteté. Michel Lauwers montre que, la sainteté ayant partie liée avec la mort depuis l'origine du culte des saints (les premiers d'entre eux ont été des martyrs) mais n'étant plus forcément associée à une mort héroïque, la scène de la mort est symboliquement essentielle dans la littérature hagiographique : "Elle conclut la présentation d'une vie sainte: l'attitude du mourant "prouve" la réalité des vertus qui lui ont été attribuées » (p. 22). Le saint doit en effet attendre la mort, sereinement pressentie, préparée par des gestes de recueillement et longuement subie dans la quiétude de la foi. La maladie, en tant qu'elle offre la possibilité d'une mort lente, joue donc un rôle essentiel dans 
exemple à la maladie qui s'empare de la fausse Guenièvre et de son proche conseiller Bertelai le Vieux, signifiant publiquement leur félonie et faisant donc éclater au grand jour le complot tramé contre la reine véritable, dans le Lancel't en prose: l'affection physique s'y double d'une terrible puanteur, antithèse de l'odeur de sainteté trahissant l'empreinte diabolique du péché ${ }^{39}$. Le corps médiéval est conçu comme une surface d'écriture symbolique où l'âme se révèle significativement, et comme "the l'cus 'f mediati $n$ between the internal-individual and the external-c'mmunal realms $s^{40}$ », ainsi que l'explique Nancy Caciola: «medieval “c'mm`n sense" tended t'view the b'dy as a material aggregate 'f the individual's identity $^{41} \gg$. De ces considérations, nous pouvons conclure que, dans la pensée médiévale chrétienne, la mutilation comme la maladie sont honteuses, parce qu'elles révèlent l'identité pécheresse de celui qui les subit : toutes deux sont l'expression publique de la maladie de l'âme. C'est dans ce contexte idéologique précis que nous pouvons comprendre la vertu thérapeutique dont Abélard revêt son émasculation, «cele tres salvable plaie » $(\mathrm{V} 79,310)$ :

Comme je travaillasse adonc touz en orgueil et en luxure, la grace de Dieu me donna neis maugré mien remede de l'une et de l'autre maladie (I 9, 233)

[...] il, par la droicturiere plaie d'une partie de mon corps, donnast medicine a nos .ii. ames

1'hagiographie. Voir Michel Lauwers, « La mort et le corps des saints. La scène de la mort dans les Vitae du haut Moyen Âge », Le M yen Âge, 94, 1988, p. 21-50.

39 « Molt longuement dura la maladie si qu'en la fin maheigna et puï si durement, quant ele comença a porrir, que nus ne la pooit soffrir.» (Lancel 't, $r{ }^{`}$ man en pr`se $d u$ XIII ${ }^{e}$ siècle, éd. Alexandre Micha, Genève, Droz, coll. "Textes littéraires français », 1978-1983, 9 t., t. I, p. 154).

${ }^{40}$ Nancy Caciola, «Mystics, Demoniacs, and the Physiology of Spirit Possession in

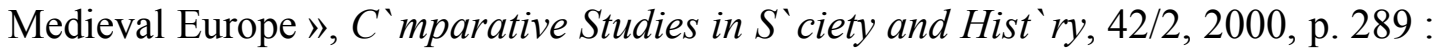
«le lieu de la médiation entre les domaines de l'internalité individuelle et de l'externalité collective » (nous traduisons).

${ }^{41}$ Ibid., p. 295 : « le "sens commun" médiéval tendait à considérer le corps comme la somme matérielle de l'identité individuelle » (nous traduisons). 


\section{(V 79, 338)}

Il empeesche la mort par plaie, il ne nous occist point ; il y met le fer pour trenchier la maladie ; il navre le corps et garist l'ame (V 85, 536)

Abélard conclut en considérant cette émasculation comme le seul remède possible, puisqu'elle élimine la cause même de la maladie morale : « la divine debonnereté ne peust pas autrement avoir mis conseil en moy fors que par entredire moy du tout ces deliz, sans nulle esperance » (V 80, 354). Abélard émasculé, c'est-à-dire symboliquement mort en tant que mari, Héloïse jouit donc des trois conditions possibles pour une femme, conformément à «la vieille classification lancée par Jérôme, reprise par Paschase Radbert, Bruno l'Écolâtre († 1101), couramment utilisée jusqu'à Thomas d'Aquin († 1274) [...]: les vierges recueilleront au centuple le fruit de leurs mérites ; les veuves, soixante fois ; les épouses, trente ${ }^{42} »$. Héloïse est à la fois vierge parce que mère spirituelle ; épouse d'Abélard d'abord, du Christ ensuite ; et doublement veuve, d'Abélard symboliquement défunt et du Christ mort sur la croix - ce qui est un lieu commun du monachisme féminin. Abélard souligne ainsi le fait que les «vestemens noirs ou vils » $(\mathrm{V} 71,54)$ de la religieuse ressemblent aux habits de la veuve en deuil pleurant son mari défunt : «Et du pleur de ces veuves sur leurs maris occis remembre l'Escripture et dist: "Femmes seans au sepulcre se complaignoient" » $(\mathrm{V} 71,58)$. Sur les « vrayes veuves », épouses éplorées du Christ, Abélard écrit encore :

Mes il [saint Paul dans la première épître à Timothée] apelle vrayes veuves toutes celles qui se sont voués a Jhesu Crist, et a qui n'est pas tant seulement mari mort, mes li mondes neis leur est crucifiéz et elles au monde; et cestes droitement convient il estre soustenues du bien de l'Eglise si

\footnotetext{
42 Jacques Dalarun, « Regards de clercs », dans Hist`ire des femmes en Occident. $V$ ' $l .2$.
} Le M'yen Âge, 'p. cit., p. 31-54, cit. p. 43. 
comme des propres rentes de leurs maris ${ }^{43}$ (VI 104, 575).

Il effleure même la dimension juridique du veuvage par une allusion à la question du droit des veuves à administrer librement ou non les fruits de leur dot, de leur douaire et des biens de leur mari ${ }^{44}$.

Le paradoxe de ce double mariage - le mariage physique avec Abélard ayant donné naissance à Astrolabe et le mariage céleste avec Jésus ayant donné naissance à des filles et fils spirituels - est exprimé par Abélard dans ce passage : «Car nous sommes une chose en Jhesu Crist, une char par la loy de mariaige: je ne cuit pas que nule chose qui soit teue me soit estrange. Mes Jhesu Crist est tiens, car tu es devenue s'espouse. » (V 86, 564). Ce double état marital ne semble pas contradictoire à Abélard. Dans ce contexte en effet, mes n'a pas le sens du mais moderne: il signifie « plus », « davantage »; « désormais », « maintenant ». Cet adverbe traduit l'addition plutôt que l'opposition : le mariage au Christ s'ajoute, succède à et surpasse le mariage avec Abélard. Il nous semble que l'émasculation d'Abélard participe de cette subtile dialectique qui fait la synthèse d'une thèse matrimoniale terrestre et de son exacte antithèse céleste.

L'attribution d'un modèle marial à une Héloïse nouvellement pure, son détournement forcé de la maternité physique au profit de l'enfantement spirituel, en l'arrachant à la classe des laboratores pour la pousser vers celle des oratores, autorise donc Abélard à demander à la jeune femme de

${ }^{43}$ Le mot mari relève de la première déclinaison des substantifs masculins (Guy Raynaud de Lage, Intr'ducti`n à l'ancien français, $2^{\mathrm{e}}$ éd. revue et corrigée par Geneviève Hasenohr, Paris, Sedes, coll. « Moyen Âge », 1993, p. 19, § 16). Ici, il est au cas régime pluriel, en fonction de complément du nom « rentes ». Or, le latin dit «sui sp `nsi», au génitif singulier. Le texte latin met donc en lumière la figure de Jésus comme unique époux des religieuses, alors que le texte français place l'accent sur la comparaison des noces célestes au mariage humain.

${ }^{44}$ Sur cette matière historique très éloignée de notre propos, nous renvoyons le lecteur à Claudia Opitz, "Contraintes et Libertés (1250-1500) », dans Hist ire des femmes en Occident. V'l. 2. Le M`yen Âge, 'p. cit., p. 277-335, cit. p. 323-328 : «Les veuves étaient-elles libres?». 
remplir son rôle d'intercession, c'est-à-dire de prier pour lui. C'est en ce sens que nous écrivions, au début de notre étude, que les intérêts d'Héloïse rejoignaient ceux du philosophe, et que la métamorphose spirituelle de l'Ève parisienne en Marie du Paraclet devait permettre l'achèvement de la rédemption d'Abélard : ce dernier, parce qu'il craint une mort qu'il croit imminente, exprime pathétiquement son besoin de la protection d'Héloïse. Et en effet, la lettre III ne constitue rien d'autre qu'un appel à la prière de l'abbesse et de ses filles pour le salut de l'âme du philosophe, tandis que la lettre $\mathrm{V}$ procède à l'envoi d'Héloïse en pèlerinage spirituel sur le tombeau du Christ, afin qu'elle rachète les péchés de l'émasculé, son époux défunt. 